\title{
EFFECTS OF SCARLET FEVER ON RHEUMATIC SUBJECTS.
}

BY

\author{
F. J. HECTOR, M.D. (Bristol),
}

Asst. Res. Medical Officer, Ham Green Hospital, Bristol.

In the practice of a large fever hospital one is constantly meeting cases of scarlet fever with a history of previous rheurnatism. In this paper an attempt is made to give a brief account of all cases of scarlet fever with cardiac complications, occurring in this hospital over a period of one year.

In all, 47 cases showing cardiac symptoms have been considered, and of these, seven had a definite history of rheumatism.

During the period observed, the percentage of scarlet fever cases which developed cardiac symptoms was $0.55 \%$, while in every case in which there was a previous history of rheumatism there was definite cardiac affection. Indeed, judging from the experience of many hundreds of cases of scarlet fever extending over a period of nearly three years, it can be stated that almost without exception an attack of scarlet fever in a rheumatic subject is followed by a reappearance of some of the symptoms associated with rheumatic infection.

The seven cases with a previous history of rheumatism will be taken first.

CASE 1. B. H. Female. Aged 10. Admitted 6.2.25. Had been an in-patient of the Bristol General Hospital two years previously suffering from wasting and chorea. The attack of scarlet fever was mild but quite typical. On admission temperature was normal, pulse 100 . There was a faint blowing systolic murmur in the aortic area, which was invariable throughout her stay in hospital. On the 32nd day of disease however there was a slight rise of temperature and the patient developed an irregular pulse and a blowing systolic murmur in the mitral area. There was no arthritis during the illness. The mitral systolic bruit had greatly diminished and the cardiac rhythm was normal when the patient was discharged 14 days later at the parents? request.

CaSe 2. C. W. Female. Aged 14. Admitted 22.5.25. This child had been admitted to the Bristol General Hospital suffering from chorea and while there had developed a rash. This was a moderate case of scarlet fever. On admission temperature was normal, pulse 76 . The cardiac sounds were normal and the point of maximum cardiac impulse was in the nipple line between the fifth and sixth ribs. Fifteen days later temperature rose to $101 \cdot \overline{2}^{\circ}$, pulse 104 . The cardiac rhythm was irregular, maximum cardiac impulse half an inch outside nipple line, with a loud, conducted apical systolic bruit. Six weeks later when the patient was discharged, the maximum cardiac impulse was in the nipple line, pulse 72 regular, and the apical systolic bruit was still well marked. 
Case 3. Female. Aged 17. Admitted 28.10.25 with mild scarlet fever. Had rheumatism twice previously. There was an occasional cardiac irregularity on admission, temperature $100^{\circ}$, pulse 104 . Fifteen days later the patient developed a faint apical systolic bruit. There was no accompanying rise of temperature. In ten days the bruit had completely disappeared.

Case 4. A. W. Male. Aged 9. Admitted 4.1.26 with scarlet fever of moderate severity. History of several attacks of chorea previously. The heart showed a diffuse heaving impulse, with the point of maximum cardiac impulse in nipple line. Soft blowing apical systolic bruit conducted towards axilla. The temperature remained normal after the first day and the cardiac signs diminished but did not disappear, pulse about 92 throughout. Twenty-six days after admission temperature rose suddenly to $102^{\circ}$, pulse $1 \overline{44}$, and the cardiac bruit became much louder and harsher. This was accompanied by a sore throat and the presence of a septic spot on the left tonsil. Six days later the patient was discharged at the request of the parents and against advice. The patient died seven days from discharge.

Case 5. L. W. Female. Aged 25. A Admitted 16.2.26, with a moderate attack of scarlet fever. Rheumatic fever three years previously. On admission temperature was normal, pulse 800 . There was a blowing diastolic murmur heard best along the left sternal border. No irregularity of rhythm. First sound fair. Twenty days after admission the temperature which had hitherto been normal commenced to vary between 97 and $99.5^{\circ}$, pulse 100 . A faint apical systolic bruit in addition to the diastolic bruit was heard. These sounds still remained when the patient was discharged three weeks later.

Case 6. L. C. Female. Aged 48. Admitted 2.3.26 with a moderate attack of scarlet fever. Had rheumatic fever 20 years before, and had no cardiac symptome since. On admission temperature $102^{\circ}$, pulse 124 (irregular). Short presystolic murmur and poor first sound. Joint pains. For 14 days there was marked irregularity of rhythm and a few creps at both bases. Seven days after admission a systolic murmur appeared. A diastolic murmur was heard a week later along the left sternal margin. At the time of discharge the general condition was good but the cardiac bruits, although fainter, still persisted. There was also less irregularity.

Case 7. E. B. Female. Aged 34. Admitted 5.3.26, fourth day of a very mild attack of scarlet fever. Rheumatic fever 18 years before. Several attacks since. Temperature normal on admission, pulse 84. Heart-heaving diffuse impulse, point of maximum impulse fifth space in nipple line. Marked presystolic murmur. Weak second sound. There was no rise of temperature during period in hospital and the cardiac murmur was slightly less marked on discharge. Pulse 88.

Of the 40 cases of scarlet fever with no previous history of rheumatisrn which developed cardiac symptoms, 12 still had symptoms on discharge. In the remainder the symptoms entirely cleared up. In quite a fair proportion of cases, therefore, scarlet fever, like rheumatism, leaves a lasting impression upon the heart. It has not been possible to ascertain whether the symptoms have disappeared after discharge. In six cases there were abnormal oardiac sounds on admission (i.e., in the early stages of scarlet fever) and it is quite possible that some of these cases had a previous rheumatic history which was not obtained or obtainable from the relatives. Seven of the 40 cases had definite arthritis. Twelve had chronically enlarged tonsils. 
In all those cases with no previous rheumatic history the symptoms were much less severe (cardiac bruit, increase of cardiac dulness, rate of respiration, etc.).

It would appear, therefore, that the almost invariable result of scarlet fever upon a heart already damaged by rheumatism is a rekindling of the old trouble which in somie cases had been quiescent for a considerable period (in Case 6, for 20 years). Further, a mild or moderate attack of scarlet fever is quite capable of bringing this about.

In view of the more than probable common streptococcal origin of the two diseases, the equally probable common port of entry of infection, the striking similarity of many of the symptoms, and their response to like treatment, it is thought that this short account of a small number of cases combining these two diseases may be of interest.

My grateful thanks are due to Dr. B. A. Peters, Senior Resident Medical Officer, Ham Green Hospital, Bristol, for permission to use the hospital records. 